\title{
Decomposition of the NVALUE constraint
}

\author{
Christian Bessiere ${ }^{1}$, George Katsirelos ${ }^{2}$, Nina Narodytska ${ }^{3}$, Claude-Guy Quimper ${ }^{4}$, \\ and Toby Walsh ${ }^{3}$ \\ 1 LIRMM, CNRS, Montpellier, email: bessiere@lirmm.fr \\ 2 CRIL-CNRS, Lens, email: gkatsi@gmail.com \\ 3 NICTA and University of NSW, Sydney, Australia, email: \\ \{nina.narodytska,toby.walsh\}@ nicta.com.au \\ 4 Université Laval, email: cquimper@gmail.com
}

\begin{abstract}
We study decompositions of the global NVALUE constraint. Our main contribution is theoretical: we show that there are propagators for global constraints like NVALUE which decomposition can simulate with the same time complexity but with a much greater space complexity. This suggests that the benefit of a global propagator may often not be in saving time but in saving space. Our other theoretical contribution is to show for the first time that range consistency can be enforced on NVALUE with the same worst-case time complexity as bound consistency. Finally, the decompositions we study are readily encoded as linear inequalities. We are therefore able to use them in integer linear programs.
\end{abstract}

\section{Introduction}

Global constraints are one of the distinguishing features of constraint programming. They capture common modelling patterns and have associated efficient propagators for pruning the search space. For example, ALL-DifFERENT is one of the best known global constraints that has proven useful in the modelling and solving of many real world problems. A number of efficient algorithms have been proposed to propagate the ALL-DifFERENT constraint (e.g. [1-3]). Whilst there is little debate that ALL-DIFFERENT is a global constraint, the formal definition of a global constraint is more difficult to pin down. One property often associated with global constraints is that they cannot be decomposed into simpler constraints without impacting either the pruning or the efficiency of propagation [4]. Recently progress has been made on the theoretical problem of understanding what is and isn't a global constraint. In particular, whilst a bound consistency propagator for the ALL-DIFFERENT constraint can be effectively simulated with a simple decomposition [5], circuit complexity lower bounds have been used to prove that a domain consistency propagator for ALL-DIFFERENT cannot be polynomially simulated by a simple decomposition [6].

In this paper, we turn to a strict generalization of the ALL-DIFFERENT constraint. NVALUE counts the number of values used by a set of variables; the ALL-DIFFERENT constraint ensures that this count equals the cardinality of the set. From a theoretical perspective, the NVALUE constraint is significantly more difficult to propagate than the ALL-DIFFERENT constraint since enforcing domain consistency is known to be NPhard [7]. Moreover, as NVALUE is a generalization of ALL-DIFFERENT, there exists no 
polynomial sized decomposition of NVALUE which achieves domain consistency [6]. Nevertheless, we show that decomposition can simulate the polynomial time algorithm for enforcing bound consistency on NVALUE but with a significant space complexity. We also prove, for the first time, that range consistency on NVALUE can be enforced in the same worst case time complexity as bound consistency. This contrasts with the ALL-DIFFERENT constraint where range consistency takes $O\left(n^{2}\right)$ time [2] but bound consistency takes just $O(n \log n)$ time [3].

The main value of these decompositions is theoretical as their space complexity is equal to their worst case time complexity. When domains are large, this space complexity may be prohibitive. In the conclusion, we argue why it appears somewhat inevitable that the space complexity is equal to the worst case time complexity. These results suggest new insight into what is and isn't a global constraint: a global constraint either provides more pruning than any polynomial sized decomposition or provides the same pruning but with lower space complexity. There are several other theoretical reasons why the decompositions studied here are interesting. First, it is technically interesting that a complex propagation algorithm like the bound consistency propagator for NVALUE can be simulated by a simple decomposition. Second, these decompositions can be readily encoded as linear inequalities and used in linear programs. In fact, we will report experiments using both constraint and integer linear programming with these decompositions. Since global constraints are one of the key differentiators between constraint and integer programming, these decompositions provide us with another tool to explore the interface between constraint and integer programming. Third, the decompositions give insights into how we might add nogood learning to a NVALUE propagator.

\section{Background}

A constraint satisfaction problem (CSP) consists of a set of variables, each with a finite domain of values, and a set of constraints. We use capitals for variables and lower case for values. We assume values are taken from the set 1 to $d$. We write $\operatorname{dom}\left(X_{i}\right)$ for the domain of possible values for $X_{i}, \min \left(X_{i}\right)$ for the smallest value in $\operatorname{dom}\left(X_{i}\right)$, $\max \left(X_{i}\right)$ for the greatest, and range $\left(X_{i}\right)$ for the interval $\left[\min \left(X_{i}\right), \max \left(X_{i}\right)\right]$. Constraint solvers typically use backtracking search to explore the space of partial assignments. After each assignment, propagation algorithms prune the search space by enforcing local consistency properties like domain, range or bound consistency. A constraint is domain consistent $(D C)$ iff when a variable is assigned any of the values in its domain, there exist compatible values in the domains of all the other variables of the constraint. Such an assignment is called a support. A CSP is domain consistent iff every constraint is domain consistent. A constraint is disentailed iff there is no possible support. A propagator which enforces domain consistency will detect disentailment, but a propagator that detects just disentailment will not enforce domain consistency. A constraint is range consistent $(R C)$ iff, when a variable is assigned any of the values in its domain, there exist compatible values between the minimum and maximum domain value for all the other variables of the constraint. Such an assignment is called a bound support. A constraint is bound consistent $(B C)$ iff the minimum and maximum value of every variable of the constraint belong to a bound support. A CSP is bound con- 
sistent iff every constraint is bound consistent. We compute the total amortized cost of enforcing a local consistency down an entire branch of the search tree. This captures the incremental cost of propagation. Finally, we will assume that a propagator is invoked at most once for each domain change and that the solver uses an optimal propagator to enforce BC on sum and channeling constraints. Such assumptions hold for modern solvers like Gecode and Ilog Solver. However, we make no assumption about the order of invocation of the constraints in a decomposition. The upper bounds we give hold regardless of the order in which constraints are processed.

A global constraint is one in which the arity of the constraint $n$ is a parameter. A decomposition of a global constraint is a CSP involving the $n$ variables of the global constraint (and possibly others), involving only constraints with fixed arity (no global constraint) or constraints that are themselves decomposable, such that the size of the CSP is polynomial in the sum of the sizes of the domains of the $n$ original variables, and such that the projection of its solutions on those $n$ variables corresponds to the solutions of the global constraint. A useful notion is algorithmic globality [4]. Informally, given a local consistency property, a global constraint is algorithmically global if there is no decomposition on which this local consistency is achieved in the same time and space complexity. We suggest here two refinements of this notion of algorithmic globality. First, we will separate the space and time complexity. That is, given a local consistency property, a global constraint is algorithmically global with respect to time (space) if there is no decomposition on which this local consistency is achieved in the same time (space) complexity. Second, unlike [4], we consider decompositions that may introduce new variables. Our results will show that, when we introduce new variables, NVALUE is not algorithmically global with respect to time but $i s$ global with respect to space.

\section{NVALUE constraint}

Pachet and Roy first proposed the NVALUE constraint [8]. Formally $\operatorname{NVAlue}\left(\left[X_{1}, \ldots, X_{n}\right], N\right)$ ensures that $N=\left|\left\{X_{i} \mid 1 \leq i \leq n\right\}\right|$. This generalizes several other global constraints including ALL-DIFFERENT (which ensures that the number of values taken by a set of variables equals the cardinality of the set) and NOT-ALL-EQUAL (which ensures a set of variables take more than one value). Enforcing domain consistency on the NVALUE constraint is NP-hard (Theorem 3 in [7]) even when $N$ is fixed (Theorem 2 in [9]). In fact, just computing the lower bound on $N$ is NP-hard (Theorem 3 in [10]). In addition, enforcing domain consistency on the NVALUE constraint is not fixed parameter tractable since it is $W$ [2]-complete [11]. However, several polynomial propagation algorithms have been proposed that achieve bound consistency and some closely related levels of local consistency $[12,9,13]$.

\subsection{Simple decomposition}

Global constraints can often be decomposed into simpler, more primitive and small arity constraints. For example, the ALL-DIFFERENT constraint can be decomposed into a quadratic number of binary inequalities. However, such decomposition often hinders propagation and can have a significant impact on the solver's ability to find solutions 
[14]. We can decompose the NVALUE constraint by introducing $0 / 1$ variables to represent which values are used and posting a sum constraint on these introduced variables:

$$
\begin{array}{cl}
X_{i}=j \rightarrow B_{j}=1 & \forall 1 \leq i \leq n, 1 \leq j \leq d \\
B_{j}=1 \rightarrow \bigvee_{i=1}^{n} X_{i}=j & \forall 1 \leq j \leq d \\
\sum_{j=1}^{d} B_{j}=N &
\end{array}
$$

Note that constraint 3 is not a fixed arity constraint, but can itself be decomposed to ternary sums without hindering bound propagation. Unfortunately, this simple decomposition hinders propagation. It can be $\mathrm{BC}$ whereas $\mathrm{BC}$ on the corresponding NVALUE constraint detects disentailment.

Theorem 1 BC on NVALUE is stronger than BC on its decomposition into (1) to (3).

Proof: Clearly BC on NVALUe is at least as strong as BC on the decomposition. To show strictness, consider $X_{1} \in\{1,2\}, X_{2} \in\{3,4\}, B_{j} \in\{0,1\}$ for $1 \leq j \leq 4$, and $N=1$. Constraints (1) to (3) are BC. However, the corresponding NVALUE constraint has no bound support and thus enforcing $\mathrm{BC}$ on it detects disentailment.

We observe that enforcing DC instead of BC on constraints (1) to (3) in the example of the proof above still does not prune any value. To decompose NVALUE without hindering propagation, we must look to more complex decompositions.

\subsection{Decomposition into AtMostNValue and AtLeastnValue}

Our first step in decomposing the NVALUE constraint is to split it into two parts: an ATMostNVALUe and an ATLEASTNVALUe constraint. AtLeastNValue $\left(\left[X_{1}, \ldots, X_{n}\right], N\right)$ holds iff $N \leq\left|\left\{X_{i} \mid 1 \leq i \leq n\right\}\right|$ whilst AtMostNValue $\left(\left[X_{1}, \ldots, X_{n}\right], N\right)$ holds iff $\left|\left\{X_{i} \mid 1 \leq i \leq n\right\}\right| \leq N$.

Running Example. Consider a NVALUE constraint over the following variables and values:

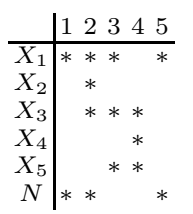

Suppose we decompose this into an ATMostNVALue and an ATLEastNVALue constraint. Consider the ATLEASTNVALUE constraint. The 5 variables can take at most 4 different values because $X_{2}, X_{3}, X_{4}$, and $X_{5}$ can only take values 2,3 and 4 . Hence, there is no bound support for $N=5$. Enforcing BC on the ATLEASTNVALUE constraint therefore prunes $N=5$. Consider now the ATMostNVALUE constraint. Since $X_{2}$ and $X_{4}$ guarantee that we take at least 2 different values, there is no bound support for $N=1$. Hence enforcing $B C$ on an ATMostNVALUE constraint prunes $N=1$. If $X_{1}=1,3$ or 5 , or $X_{5}=3$ then any complete assignment uses at least 3 different values. Hence there is also no bound support for these assignments. Pruning these values 
gives bound consistent domains for the original NVALUE constraint:

\begin{tabular}{|c|c|}
\hline$\overline{X_{1}}$ & $*$ \\
\hline$X_{2}$ & 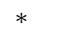 \\
\hline $\begin{array}{c}X_{3} \\
X_{4}\end{array} \mid$ & $* * *$ \\
\hline $\begin{array}{l}X_{5} \\
x_{1}\end{array}$ & \\
\hline$N$ & \\
\hline
\end{tabular}

To show that decomposing the NVALUE constraint into these two parts does not hinder propagation in general, we will use the following lemma. Given an assignment $S$ of values, $\operatorname{card}(S)$ denotes the number of distinct values in $S$. Given a vector of variables $X=X_{1} \ldots X_{n}, \operatorname{card}_{\uparrow}(X)=\max \left\{\operatorname{card}(S) \mid S \in \Pi_{X_{i} \in X} \operatorname{range}\left(X_{i}\right)\right\}$ and $\operatorname{card}_{\downarrow}(X)=\min \left\{\operatorname{card}(S) \mid S \in \Pi_{X_{i} \in X} \operatorname{range}\left(X_{i}\right)\right\}$.

Lemma 1 (adapted from [13]) Consider $\operatorname{NValue}\left(\left[X_{1}, \ldots, X_{n}\right], N\right)$. If $\operatorname{dom}(N) \subseteq$ $\left[\operatorname{card}_{\downarrow}(X), \operatorname{card}_{\uparrow}(X)\right]$, then the bounds of $N$ have bound supports.

Proof: Let $S_{\min }$ be an assignment of $X$ in $\Pi_{X_{i} \in X} \operatorname{range}\left(X_{i}\right)$ with $\operatorname{card}\left(S_{\min }\right)=$ $\operatorname{card}_{\downarrow}(X)$ and $S_{\text {max }}$ be an assignment of $X$ in $\Pi_{X_{i} \in X} \operatorname{range}\left(X_{i}\right)$ with $\operatorname{card}\left(S_{\max }\right)=$ $\operatorname{card}_{\uparrow}(X)$. Consider the sequence $S_{\text {min }}=S_{0}, S_{1}, \ldots, S_{n}=S_{\text {max }}$ where $S_{k+1}$ is the same as $S_{k}$ except that $X_{k+1}$ has been assigned its value in $S_{\text {max }}$ instead of its value in $S_{\text {min }} \cdot\left|\operatorname{card}\left(S_{k+1}\right)-\operatorname{card}\left(S_{k}\right)\right| \leq 1$ because they only differ on $X_{k+1}$. Hence, for any $p \in\left[\operatorname{card}_{\downarrow}(X), \operatorname{card}_{\uparrow}(X)\right]$, there exists $k \in 1 . . n$ with $\operatorname{card}\left(S_{k}\right)=p$. Thus, $\left(S_{k}, p\right)$ is a bound support for $p$ on $\operatorname{NVALUE}\left(\left[X_{1}, \ldots, X_{n}\right], N\right)$. Therefore, $\min (N)$ and $\max (N)$ have a bound support.

We now prove that decomposing the NVALue constraint into ATMostNVALUe and ATLEASTNVALUE constraints does not hinder pruning when enforcing BC.

Theorem $2 B C$ on $\operatorname{NValue}\left(\left[X_{1}, \ldots, X_{n}\right], N\right)$ is equivalent to $B C$ on AtMostNValue $\left(\left[X_{1}, \ldots, X_{n}\right], N\right)$ and on AtLeastNValue $\left(\left[X_{1}, \ldots, X_{n}\right], N\right)$.

Proof: Suppose the AtMostNValue and AtLeastNValue constraints are BC. The AtMostNValue constraint guarantees that $\operatorname{card}_{\downarrow}(X) \leq \min (N)$ and the ATLEastNVALue constraint guarantees that $\operatorname{card}_{\uparrow}(X) \geq \max (N)$. Therefore, $\operatorname{dom}(N) \in\left[\operatorname{card}_{\downarrow}(X), \operatorname{card}_{\uparrow}(X)\right]$. By Lemma 1, the variable $N$ is bound consistent.

Consider a variable/bound value pair $X_{i}=b$. Let $\left(S_{\text {least }}^{b}, p_{1}\right)$ be a bound support of $X_{i}=b$ in the ATLEASTNVALUE constraint and $\left(S_{\text {most }}^{b}, p_{2}\right)$ be a bound support of $X_{i}=b$ in the ATMostNVALUE constraint. We have $\operatorname{card}\left(S_{\text {least }}^{b}\right) \geq p_{1}$ and $\operatorname{card}\left(S_{\text {most }}^{b}\right) \leq p_{2}$ by definition of AtLeastNVAlue and AtMostNVALue. Consider the sequence $S_{\text {least }}^{b}=S_{0}^{b}, S_{1}^{b}, \ldots, S_{n}^{b}=S_{\text {most }}^{b}$ where $S_{k+1}^{b}$ is the same as $S_{k}^{b}$ except that $X_{k+1}$ has been assigned its value in $S_{\text {most }}^{b}$ instead of its value in $S_{\text {least }}^{b} .\left|\operatorname{card}\left(S_{k+1}^{b}\right)-\operatorname{card}\left(S_{k}^{b}\right)\right| \leq 1$ because they only differ on $X_{k+1}$. Hence, there exists $k \in 1 . . n$ with $\min \left(p_{1}, p_{2}\right) \leq \operatorname{card}\left(S_{k}^{b}\right) \leq \max \left(p_{1}, p_{2}\right)$. We know that $p_{1}$ and $p_{2}$ belong to range $(N)$ because they belong to bound supports. Thus, $\operatorname{card}\left(S_{k}^{b}\right) \in \operatorname{range}(N)$ and $\left(S_{k}^{b}, \operatorname{card}\left(S_{k}^{b}\right)\right)$ is a bound support for $X_{i}=b$ on $\operatorname{NVALUE}\left(\left[X_{1}, \ldots, X_{n}\right], N\right)$.

When enforcing domain consistency, Bessiere et al. [13] noted that decomposing the NVALue constraint into AtMostNVALUe and AtLeastNVAlue constraints 
does hinder propagation, but only when $\operatorname{dom}(N)$ contains just $\operatorname{card}_{\downarrow}(X)$ and $\operatorname{card}_{\uparrow}(X)$ and there is a gap in the domain in-between (see Theorem 1 in [13] and the discussion that follows). When enforcing $\mathrm{BC}$, any such gap in the domain for $N$ is ignored.

\section{AtMostnValue constraint}

We now give a decomposition for the ATMOSTNVALUE constraint which does not hinder bound consistency propagation. To decompose the ATMOSTNVALUE constraint, we introduce $0 / 1$ variables, $A_{i l u}$ to represent whether $X_{i}$ uses a value in the interval $[l, u]$, and "pyramid" variables, $M_{l u}$ with domains $[0, \min (u-l+1, n)]$ which count the number of values taken inside the interval $[l, u]$. To constrain these introduced variables, we post the following constraints:

$$
\begin{array}{cl}
A_{i l u}=1 \Longleftrightarrow X_{i} \in[l, u] & \forall 1 \leq i \leq n, 1 \leq l \leq u \leq d \\
A_{i l u} \leq M_{l u} & \forall 1 \leq i \leq n, 1 \leq l \leq u \leq d \\
M_{1 u}=M_{1 k}+M_{(k+1) u} & \forall 1 \leq k<u \leq d \\
M_{1 d} \leq N &
\end{array}
$$

Running Example. Consider the decomposition of an ATMostNVALue constraint over the following variables and values:

$$
\begin{array}{r|rrrrr} 
& 1 & 2 & 3 & 4 & 5 \\
\hline X_{1} & * & * & * & * \\
X_{2} & * & & & \\
X_{3} & * & * & * & \\
X_{4} & \multicolumn{3}{|c}{*} & * \\
X_{5} & \multicolumn{2}{|c}{*} & * \\
N & * & * & &
\end{array}
$$

Observe that we consider that value 5 for $N$ has already been pruned by AtLeastNVAlue, as will be shown in next sections. Bound consistency reasoning on the decomposition will make the following inferences. As $X_{2}=2$, from (4) we get $A_{222}=1$. Hence by (5), $M_{22}=1$. Similarly, as $X_{4}=4$, we get $A_{444}=1$ and $M_{44}=1$. Now $N \in\{1,2\}$. By (7) and (6), $M_{15} \leq N, M_{15}=M_{14}+M_{55}$, $M_{14}=M_{13}+M_{44}, M_{13}=M_{12}+M_{33}, M_{12}=M_{11}+M_{22}$. Since $M_{22}=M_{44}=1$, we deduce that $N>1$ and hence $N=2$. This gives $M_{11}=M_{33}=M_{55}=0$. By (5), $A_{111}=A_{133}=A_{155}=A_{533}=0$. Finally, from (4), we get $X_{1}=2$ and $X_{5}=3$. This gives us bound consistent domains for the ATMOSTNVALUE constraint.

We now prove that this decomposition does not hinder propagation in general.

Theorem 3 BC on constraints (4) to (7) is equivalent to BC on ATMostNVALUe $\left(\left[X_{1}, \ldots, X_{n}\right], N\right)$, and takes $O\left(n d^{3}\right)$ time to enforce down the branch of the search tree.

Proof: First note that changing the domains of the $X$ variables cannot affect the upper bound of $N$ by the ATMostNVALue constraint and, conversely, changing the lower bound of $N$ cannot affect the domains of the $X$ variables. 
Let $Y=\left\{X_{p_{1}}, \ldots, X_{p_{k}}\right\}$ be a maximum cardinality subset of variables of $X$ whose ranges are pairwise disjoint (i.e., range $\left(X_{p_{i}}\right) \cap \operatorname{range}\left(X_{p_{j}}\right)=\emptyset, \forall i, j \in 1 . . k, i \neq j$ ). Let $I_{Y}=\left\{\left[b_{i}, c_{i}\right] \mid b_{i}=\min \left(X_{p_{i}}\right), c_{i}=\max \left(X_{p_{i}}\right), X_{p_{i}} \in Y\right\}$ be the corresponding ordered set of disjoint ranges of the variables in $Y$. It has been shown in [9] that $|Y|=$ $\operatorname{card}_{\downarrow}(X)$.

Consider the interval $\left[b_{i}, c_{i}\right] \in I_{Y}$. Constraints (5) ensure that the variables $M_{b_{i} c_{i}}$ $i=[1, \ldots, k]$ are greater than or equal to 1 and constraints (6) ensure that the variable $M_{1 d}$ is greater than or equal to the sum of lower bounds of variables $M_{b_{i} c_{i}}$, $i=[1, \ldots, k]$, because intervals $\left[b_{i}, c_{i}\right]$ are disjoint. Therefore, the variable $N$ is greater than or equal to $\operatorname{card}_{\downarrow}(X)$ and it is bound consistent.

We show that when $N$ is BC and $\operatorname{dom}(N) \neq\left\{\operatorname{card}_{\downarrow}(X)\right\}$, all $X$ variables are $B C$. Take any assignment $S \in \Pi_{X_{i} \in X} \operatorname{range}\left(X_{i}\right)$ such that $\operatorname{card}(S)=\operatorname{card}_{\downarrow}(X)$. Let $S\left[X_{i} \leftarrow b\right]$ be the assignment $S$ where the value of $X_{i}$ in $S$ has been replaced by $b$, one of the bounds of $X_{i}$. We know that $\operatorname{card}\left(S\left[X_{i} \leftarrow b\right]\right) \in[\operatorname{card}(S)-1, \operatorname{card}(S)+1]=$ $\left[\operatorname{card}_{\downarrow}(X)-1, \operatorname{card}_{\downarrow}(X)+1\right]$ because only one variable has been flipped. Hence, any assignment $(S, p)$ with $p \geq \operatorname{card}_{\downarrow}(X)+1$ is a bound support. $\operatorname{dom}(N)$ necessarily contains such a value $p$ by assumption.

The only case when pruning might occur is if the variable $N$ is ground and $\operatorname{card}_{\downarrow}(X)=N$. Constraints (6) imply that $M_{1 d}$ equals the sum of variables $M_{1, b_{1}-1}+$ $M_{b_{1}, c_{1}}+M_{c_{1}+1, b_{2}-1} \ldots+M_{b_{N}, c_{N}}+M_{c_{N}+1, d}$. The lower bound of the variable $M_{c_{i}, b_{i}}$ is greater than one and there are $|Y|=\operatorname{card}_{\downarrow}(X)=N$ of these intervals. Therefore, by constraint (7), the upper bound of variables $M_{c_{i-1}+1, b_{i}-1}$ that correspond to intervals outside the set $I_{Y}$ are forced to zero.

There are $O\left(n d^{2}\right)$ constraints (4) and constraints (5) that can be woken $O(d)$ times down the branch of the search tree. Each requires $O(1)$ time for a total of $O\left(n d^{3}\right)$ down the branch. There are $O\left(d^{2}\right)$ constraints (6) which can be woken $O(n)$ times down the branch and each invocation takes $O(1)$ time. This gives a total of $O\left(n d^{2}\right)$. The final complexity down the branch of the search tree is therefore $O\left(n d^{3}\right)$.

The proof of theorem 3 also provides the corollary that enforcing range on consistency on constraints 4 enforces range consistency on ATMOSTNVALUE. Note that theorem 3 shows that the BC propagator of ATMOSTNVALUE [12] is not algorithmically global with respect to time, as $\mathrm{BC}$ can be achieved with a decomposition with comparable time complexity. On the other hand, the $O\left(n d^{2}\right)$ space complexity of this decomposition suggests that it is algorithmically global with respect to space. Of course, we only provide upper bounds here, so it may be that ATMOSTNVALUE is not algorithmically global with respect to either time or space.

\section{Faster decompositions}

We can improve how the solver handles this decomposition of the ATMostNVALUE constraint by adding implied constraints and by implementing specialized propagators. Our first improvement is to add an implied constraint and enforce BC on it:

$$
M_{1 d}=\sum_{i=1}^{d} M_{i i}
$$


This does not change the asymptotic complexity of reasoning with the decomposition, nor does it improve the level of propagation achieved. However, we have found that the fixed point of propagation is reached quicker in practice with such an implied constraint.

Our second improvement decreases the asymptotic complexity of enforcing BC on the decomposition of Section 4. The complexity is dominated by reasoning with constraints (4) which channel from $X_{i}$ to $A_{i l u}$ and thence onto $M_{l u}$ (through constraints (5)). If constraints (4) are not woken uselessly, enforcing BC costs $O(1)$ per constraint down the branch. Unfortunately, existing solvers wake up such constraints as soon as a bound is modified, thus giving a cost in $O(d)$. We therefore implemented a specialized propagator to channel between $X_{i}$ and $M_{l u}$ efficiently. To be more precise, we remove the $O\left(n d^{2}\right)$ variables $A_{i l u}$ and replace them with $O(n d)$ Boolean variables $Z_{i j}$. We then add the following constraints

$$
\begin{array}{rlr}
Z_{i j}=1 \Longleftrightarrow X_{i} \leq j & 1 \leq j \leq d \\
Z_{i(l-1)}=1 \vee Z_{i u}=0 \vee M_{l u}>0 & 1 \leq l \leq u \leq d, 1 \leq i \leq n
\end{array}
$$

These constraints are enough to channel changes in the bounds of the $X$ variables to $M_{l u}$. There are $O(n d)$ constraints (9), each of which can be propagated in time $O(d)$ over a branch, for a total of $O\left(n d^{2}\right)$. There are $O\left(n d^{2}\right)$ clausal constraints (10) and each of them can be made $\mathrm{BC}$ in time $O(1)$ down a branch of the search tree, for a total cost of $O\left(n d^{2}\right)$. Since channeling dominates the asymptotic complexity of the entire decomposition of Section 4, this improves the complexity of this decomposition to $O\left(n d^{2}\right)$. This is similar to the technique used in [5] to improve the asymptotic complexity of the decomposition of the ALL-DIFFERENT constraint.

Our third improvement is to enforce stronger pruning by observing that when $M_{l u}=0$, we can remove the interval $[l, u]$ from all variables, regardless of whether this modifies their bounds. This corresponds to enforcing RC on constraints (4). Interestingly, this is sufficient to achieve RC on the ATMOSTNVALUE constraint. Unfortunately, constraints (10) cannot achieve this pruning and using constraints (4) increases the complexity of the decomposition back to $O\left(n d^{3}\right)$. Instead we extend the decomposition with $O(d \log d)$ Boolean variables $B_{i l\left(l+2^{k}\right)} \in[0,1], 1 \leq i \leq n, 1 \leq l \leq d, 0 \leq$ $k \leq\lfloor\log d\rfloor$. The following constraint ensures that $B_{i j j}=1 \Longleftrightarrow X_{i}=j$.

$$
\text { DomainBitmap }\left(X_{i},\left[B_{i 11}, \ldots, B_{i d d}\right]\right)
$$

Clearly we can enforce RC on this constraint in time $O(d)$ over a branch, and $O(n d)$ for all variables $X_{i}$. We can then use the following clausal constraints to channel from variables $M_{l u}$ to these variables and on to the $X$ variables. These constraints are posted for every $1 \leq i \leq n, 1 \leq l \leq u \leq d, 1 \leq j \leq d$ and integers $k$ such that $0 \leq k \leq$ $\lfloor\log d\rfloor$ :

$$
\begin{aligned}
B_{i j\left(j+2^{k+1}-1\right)} & =1 \vee B_{i j\left(j+2^{k}-1\right)}=0 & & \\
B_{i j\left(j+2^{k+1}-1\right)} & =1 \vee B_{i\left(j+2^{k}\right)\left(j+2^{k+1}-1\right)}=0 & & \\
M_{l u} & \neq 0 \vee B_{i l\left(l+2^{k}-1\right)}=0 & & 2^{k} \leq u-l+1<2^{k+1} \\
M_{l u} & \neq 0 \vee B_{i\left(u-2^{k}+1\right) u}=0 & & 2^{k} \leq u-l+1<2^{k+1}
\end{aligned}
$$


The variable $B_{i l\left(l+2^{k}-1\right)}$, similarly to the variables $A_{l u}$, is true when $X_{i} \in[l, l+$ $\left.2^{k}-1\right]$, but instead of having one such variable for every interval, we only have them for intervals whose length is a power of two. When $M_{l u}=0$, with $2^{k} \leq u-l+1<2^{k+1}$, the constraints (14)-(15) set to 0 the $B$ variables that correspond to the two intervals of length $2^{k}$ that start at $l$ and finish at $u$, respectively. In turn, the constraints (12)-(13) set to 0 the $B$ variables that correspond to intervals of length $2^{k-1}$, all the way down to intervals of size 1 . These trigger the constraints (11), so all values in the interval $[l, u]$ are removed from the domains of all variables.

Example. Suppose $X_{1} \in[5,9]$. Then, by (9), $Z_{14}=0, Z_{19}=1$ and by (10), $M_{59}>0$. Conversely, suppose $M_{59}=0$ and $X_{1} \in[1,10]$. Then, by (14)-(15), we get $B_{158}=0$ and $B_{169}=0$. From $B_{158}=0$ and (12)-(13) we get $B_{156}=0, B_{178}=0, B_{155}=$ $B_{166}=B_{177}=B_{188}=0$, and by (11), the interval $[5,8]$ is pruned from $X_{1}$. Similarly, $B_{169}=0$ causes the interval $[6,9]$ to be removed from $X_{1}$, so $X_{1} \in[1,4] \cup\{10\}$.

Note that RC can be enforced on each of these constraints in constant time over a branch. There exist $O(n d \log d)$ of the constraints (12)-(13) and $O\left(n d^{2}\right)$ of the constraints (14)-(15), so the total time to propagate them all down a branch is $O\left(n d^{2}\right)$.

\section{AtLeastNValue constraint}

There is a similar decomposition for the ATLEASTNVALUE constraint. We introduce $0 / 1$ variables, $A_{i l u}$ to represent whether $X_{i}$ uses a value in the interval $[l, u]$, and integer variables, $E_{l u}$ with domains $[0, n]$ to count the number of times values in $[l, u]$ are $r e$ used, that is, how much the number of variables taking values in $[l, u]$ exceeds the number $u-l+1$ of values in $[l, u]$. To constrain these introduced variables, we post the following constraints:

$$
\begin{array}{cl}
A_{i l u}=1 \Longleftrightarrow X_{i} \in[l, u] & \forall 1 \leq i \leq n, 1 \leq l \leq u \leq d \\
E_{l u} \geq \sum_{i=1}^{n} A_{i l u}-(u-l+1) & \forall 1 \leq l \leq u \leq d \\
E_{1 u}=E_{1 k}+E_{(k+1) u} & \forall 1 \leq k<u \leq d \\
N \leq n-E_{1 d} &
\end{array}
$$

Running Example. Consider the decomposition of an ATLEASTNVALUE constraint over the following variables and values:

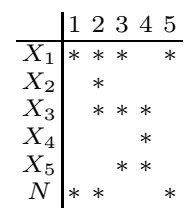

Bound consistency reasoning on the decomposition will make the following inferences. As $\operatorname{dom}\left(X_{i}\right) \subseteq[2,4]$ for $i \in 2 . .5$, from (16) we get $A_{i 24}=1$ for $i \in 2 . .5$. Hence, by (17), $E_{24} \geq 1$. By (18), $E_{15}=E_{14}+E_{55}, E_{14}=E_{11}+E_{24}$. Since $E_{24} \geq 1$ we deduce that $E_{15} \geq 1$. Finally, from (19) and the fact that $n=5$, we get $N \leq 4$. This gives us bound consistent domains for the ATLEASTNVALUE constraint. 
We now prove that this decomposition does not hinder propagation in general.

Theorem $4 B C$ on the constraints (16) to (19) is equivalent to $B C$ on AtLeastNValue $\left(\left[X_{1}, \ldots, X_{n}\right], N\right)$, and takes $O\left(n d^{3}\right)$ time to enforce down the branch of the search tree.

Proof: First note that changing the domains of the $X$ variables cannot affect the lower bound of $N$ by the ATLEASTNVALUE constraint and, conversely, changing the upper bound of $N$ cannot affect the domains of the $X$ variables.

It is known [12] that $\operatorname{card}_{\uparrow}(X)$ is equal to the size of a maximum matching $M$ in the value graph of the constraint. Since $N \leq n-E_{1 d}$, we show that the lower bound of $E_{1 d}$ is equal to $n-|M| .^{5}$ We first show that we can construct a matching $M(E)$ of size $n-\min \left(E_{1 d}\right)$, then show that it is a maximum matching. The proof uses a partition of the interval $[1, d]$ into a set of maximal saturated intervals $I=\left\{\left[b_{j}, c_{j}\right]\right\}, j=1, \ldots, k$ such that $\min \left(E_{b_{j}, c_{j}}\right)=\sum_{i=1}^{n} \min \left(A_{i b_{j} c_{j}}\right)-\left(c_{j}-b_{j}+1\right)$ and a set of unsaturated intervals $\left\{\left[b_{j}, c_{j}\right]\right\}$ such that $\min \left(E_{b_{j}, c_{j}}\right)=0$.

Let $I=\left\{\left[b_{j}, c_{j}\right] \mid j \in[1 \ldots k]\right\}$ be the ordered set of maximal intervals such that $\min \left(E_{b_{j}, c_{j}}\right)=\sum_{i=1}^{n} \min \left(A_{i b_{j} c_{j}}\right)-\left(c_{j}-b_{j}+1\right)$. Note that the intervals in $I$ are disjoint otherwise intervals are not maximal. An interval $\left[b_{i}, c_{i}\right]$ is smaller than $\left[b_{j}, c_{j}\right]$ iff $c_{i}<b_{j}$. We denote the union of the first $j$ intervals $D_{I}^{j}=\bigcup_{i=1}^{j}\left[b_{i}, c_{i}\right]$, $j=[1, \ldots, k], p=\left|D_{I}^{k}\right|$ and the variables whose domain is inside one of intervals $I$ $X_{I}=\left\{X_{p_{i}} \mid \operatorname{dom}\left(X_{p_{i}}\right) \subseteq D_{I}^{k}\right\}$.

Our construction of a matching uses two sets of variables, $X_{I}$ and $X \backslash X_{I}$. First, we identify the cardinality of these two sets. Namely, we show that the size of the set $X_{I}$ is $p+\min \left(E_{1, d}\right)$ and the size of the set $X \backslash X_{I}$ is $n-\left(p+\min \left(E_{1, d}\right)\right)$.

Intervals $I$ are saturated therefore each value from these intervals are taken by a variable in $X_{I}$. Therefore, $X_{I}$ has size at least $p$. Moreover, there exist $\min \left(E_{1 d}\right)$ additional variables that take values from $D_{I}^{k}$, because values from intervals between two consecutive intervals in $I$ do not contribute to the lower bound of the variable $E$ by construction of $I$. Therefore, the number of variables in $D_{I}^{k}$ is at least $p+\min \left(E_{1, d}\right)$. Note that constraints (18) imply that $E_{1 d}$ equals the sum of variables $E_{1, b_{1}-1}+E_{b_{1}, c_{1}}+E_{c_{1}+1, b_{2}-1} \ldots+E_{b_{k}, c_{k}}+E_{c_{k}+1, d}$. As intervals in $I$ are disjoint then $\sum_{i=1}^{k} \min \left(E_{b_{i}, c_{i}}\right)=\left|X_{I}\right|-p$. If $\left|X_{I}\right|>p+\min \left(E_{1, d}\right)$ then $\sum_{i=1}^{k} \min \left(E_{b_{i}, c_{i}}\right)>\min \left(E_{1, d}\right)$ and the lower bound of the variable $E_{1 d}$ will be increased. Hence, $\left|X_{I}\right|=p+\min \left(E_{1, d}\right)$.

Since all these intervals are saturated, we can construct a matching $M_{I}$ of size $p$ using the variables in $X_{I}$. The size of $X \backslash X_{I}$ is $n-p-\min \left(E_{1 d}\right)$. We show by contradiction that we can construct a matching $M_{D-D_{I}^{k}}$ of size $n-p-\min \left(E_{1 d}\right)$ using the variables in $X \backslash X_{I}$ and the values $D-D_{I}^{k}$.

Suppose such a matching does not exist. Then, there exists an interval $[b, c]$ such that $\left|\left(D \backslash D_{I}^{k}\right) \cap[b, c]\right|<\sum_{i \in X \backslash X_{I}} \min \left(A_{i b c}\right)$, i.e., after consuming the values in $I$ with variables in $X_{I}$, we are left with fewer values in $[b, c]$ than variables whose domain is contained in $[b, c]$. We denote $p^{\prime}=\left|[b, c] \cap D_{I}^{k}\right|$, so that $p^{\prime}$ is the number of values inside the interval $[b, c]$ that are taken by variables in $X_{I}$. The total number of

\footnotetext{
${ }^{5}$ We assume that $E_{1 d}$ is not pruned by other constraints.
} 
variables inside the interval $[b, c]$ is greater than or equal to $\sum_{i=1}^{n} \min \left(A_{i b c}\right)$. The total number of variables $X_{I}$ inside the interval $[b, c]$ equals to $p^{\prime}+\min \left(E_{b, c}\right)$. Therefore, $\sum_{i \in X \backslash X_{I}} \min \left(A_{i b c}\right) \leq \sum_{i=1}^{n} \min \left(A_{i b c}\right)-p^{\prime}-\min \left(E_{b, c}\right)$. On the other hand, the number of values that are not taken by the variables $X_{I}$ in the interval $[b, c]$ is $c-$ $b+1-p^{\prime}$. Therefore, we obtain the inequality $c-b+1-p^{\prime}<\sum_{i=1}^{n} \min \left(A_{i b c}\right)-$ $p^{\prime}-\min \left(E_{b, c}\right)$ or $\min \left(E_{b c}\right)<\sum_{i=1}^{n} \min \left(A_{i b c}\right)-(c-b+1)$. By construction of $I, \sum_{i=1}^{n} \min \left(A_{i b c}\right)-(c-b+1)<\min \left(E_{b c}\right)$, otherwise the intervals in $I$ that are subsets of $[b, c]$ are not maximal. This leads to a contradiction, so we can construct a matching $M(E)$ of size $n-\min \left(E_{1 d}\right)$.

Now suppose that $M(E)$ is not a maximum matching. This means that $\min \left(E_{1 d}\right)$ is overestimated by propagation on (16) and (19). Since $M(E)$ is not a maximum matching, there exists an augmenting path of $M(E)$, that produces $M^{\prime}$, such that $\left|M^{\prime}\right|=|M(E)|+1$. This new matching covers all the values that $M(E)$ covers and one additional value $q$. We show that $q$ cannot belong to the interval $[1, d]$.

The value $q$ cannot be in any interval in $I$, because all values in $\left[b_{i}, c_{i}\right] \in I$ are used by variables whose domain is contained in $\left[b_{i}, c_{i}\right]$. In addition, $q$ cannot be in an interval $[b, c]$ between two consecutive intervals in $I$, because those intervals do not contribute to the lower bound of $E_{1 d}$. Thus, $M^{\prime}$ cannot cover more values than $M(E)$ and they must have the same size, a contradiction.

We show that when $N$ is $\mathrm{BC}$ and $\operatorname{dom}(N) \neq\left\{\operatorname{card}_{\uparrow}(X)\right\}$, all $X$ variables are $B C$. Take any assignment $S \in \Pi_{X_{i} \in X} \operatorname{range}\left(X_{i}\right)$ such that $\operatorname{card}(S)=\operatorname{card}_{\uparrow}(X)$. Let $S\left[X_{i} \leftarrow b\right]$ be the assignment $S$ where the value of $X_{i}$ in $S$ has been replaced by $b$, one of the bounds of $X_{i}$. We know that $\operatorname{card}\left(S\left[X_{i} \leftarrow b\right]\right) \in[\operatorname{card}(S)-1, \operatorname{card}(S)+1]=$ $\left[\operatorname{card}_{\uparrow}(X)-1, \operatorname{card}_{\uparrow}(X)+1\right]$ because only one variable has been flipped. Hence, any assignment $(S, p)$ with $p \leq \operatorname{card}_{\uparrow}(X)-1$ is a bound support. $\operatorname{dom}(N)$ necessarily contains such a value $p$ by assumption.

We now show that if $N=\operatorname{card}_{\uparrow}(X)$, enforcing BC on the constraints (16)-(19) makes the variables $X$ BC with respect to the ATLEastNVAlue constraint. We first observe that in a bound support, variables $X$ must take the maximum number of different values because $N=\operatorname{card}_{\uparrow}(X)$. Hence, in a bound support, variables $X$ that are not included in a saturated interval will take values outside any saturated interval they overlap and they all take different values. We recall that $\min \left(E_{1 d}\right)=n-|M|=$ $n-\operatorname{card}_{\uparrow}(X)$. Hence, by constraint (19), $E_{1 d}=n-N$. We recall the the size of set $X_{I}$ equals $p+E_{1 d}$. Constraints (18) imply that $E_{1 d}$ equals the sum of variables $E_{1, b_{1}-1}+E_{b_{1}, c_{1}}+E_{c_{1}+1, b_{2}-1} \ldots+E_{b_{k}, c_{k}}+E_{c_{k}+1, d}$ and $\sum_{i=1}^{k} \min \left(E_{b_{i}, c_{i}}\right)=$ $\left|X_{I}\right|-p=\min \left(E_{1 d}\right)=\max \left(E_{1 d}\right)$. Hence, by constraints (18), the upper bounds of all variables $E_{b_{i}, c_{i}}$ that correspond to the saturated intervals are forced to $\min \left(E_{b_{i}, c_{i}}\right)$. Thus, by constraints (16) and (17), all variables in $X \backslash X_{I}$ have their bounds pruned if they belong to $D_{I}^{k}$. By constraints (18) again, the upper bounds of all variables $E_{l u}$ that correspond to the unsaturated intervals are forced to take value 0 , and all variables $E_{l^{\prime} u^{\prime}}$ with $\left[l^{\prime}, u^{\prime}\right] \subseteq[l, u]$ are forced to 0 as well. Thus, by constraints (16) and (17), all variables in $X \backslash X_{I}$ have their bounds pruned if they belong to a Hall interval of other variables in $X \backslash X_{I}$. This is what BC on the ALL-DIFFERENT constraint does [5].

There are $O\left(n d^{2}\right)$ constraints (16) that can be woken $O(d)$ times down the branch of the search tree in $O(1)$, so a total of $O\left(n d^{3}\right)$ down the branch. There are $O\left(d^{2}\right)$ 
constraints (17) which can be propagated in time $O(n)$ down the branch for a $O\left(n d^{2}\right)$. There are $O\left(d^{2}\right)$ constraints (18) which can be woken $O(n)$ times each down the branch for a total cost in $O(n)$ time down the branch. Thus a total of $O\left(n d^{2}\right)$. The final complexity down the branch of the search tree is therefore $O\left(n d^{3}\right)$.

The complexity of enforcing BC on ATLEASTNVALUE can be improved to $O\left(n d^{2}\right)$ in a way similar to that described in Section 5 and in [5]. As with ATMOSTNVALUE, enforcing RC on constraints (16) enforces RC on ATLEASTNVALUE, but in this case we cannot reduce the complexity below $O\left(n d^{3}\right)$. Similarly to ATMostNVALue, theorem 4 shows that the bound consistency propagator of ATLEASTNVALUE is not algorithmically global with respect to time and provides evidence that it is algorithmically global with respect to space.

\section{Experimental results}

As noted before, the main value of these decompositions is theoretical: demonstrating that the bound consistency propagator of [12] for the NVALUE constraint can be simulated using a simple decomposition with comparable time complexity over a branch of the search tree but greater space complexity. To see when this space complexity hits, we performed some experiments. We used a benchmark problem, the dominating set of the Queen's graph used in previous studies of NVALUE [13] and ran experiments with Ilog Solver 6.2 and Ilog CPLEX 9.1 on an Intel Xeon 4 CPU, 2.0 Ghz, 4Gb RAM. The dominating set of the Queen's graph problem is to put the minimum number of queens on a $n \times n$ chessboard, so that each square either contains a queen or is attacked by one. This is equivalent to the dominating set problem of the Queen's graph. Each vertex in the Queen's graph corresponds to a square of the chessboard and there exists an edge between two vertices iff a queen from one square can attack a queen from the other square. To model the problem, we use a variable $X_{i}$ for each square, and values from 1 to $n^{2}$ and post a single AtMostNValue $\left(\left[X_{1}, \ldots, X_{n^{2}}\right], N\right)$ constraint. The value $j$ belongs to $\operatorname{dom}\left(X_{i}\right)$ iff there exists an edge $(i, j)$ in the Queen's graph or $j=i$. For $n \leq 120$, all minimum dominating sets for the Queen's problem are either of size $\lceil n / 2\rceil$ or $\lceil n / 2+1\rceil[15]$. We therefore only solved instances for these two values of $N$.

We compare our decomposition with the simple decomposition of the ATMostNVALue constraint in Ilog Solver and Ilog CPLEX solvers. The simple decomposition is the one described in Section 3.1 except that in constraint (3), we replace "=" by " $\leq$ ". We denote this decomposition Occs and Occs ${ }^{C L E X}$ in Ilog Solver and CPLEX, respectively. To encode this decomposition into an integer linear program, we introduce literals $b_{i j}, i, j \in\left[1, n^{2}\right]$ and use a direct encoding with $b_{i j}$ for the truth of $X_{i}=j$ and channeling inequalities $1-b_{i j}+B_{j} \geq 1, i, j \in\left[1, n^{2}\right]$. We use the direct encoding of variables domains to avoid using logic constraints, like disjunction and implication constraints in CPLEX. The default transformation of logic constraints in CPLEX appears to generate large ILP models and this slows down the search.

The BC decomposition is described in Section 4, which we call Pyramid $B C$ and Pyramid $C P C E X$ in Ilog Solver and CPLEX, respectively. In Ilog Solver, as explained in Section 5, we channel the variables $X_{i}$ directly to the pyramid variables $M_{l u}$ to avoid introducing many auxiliary variables $A_{i l u}$ and we add the redundant constraint 
$\sum_{i=1}^{n^{2}} M_{i i}=M_{1, n^{2}}$ to the decomposition to speed up the propagation across the pyramid. We re-implemented the ternary sum constraint in Ilog for a 30\% speedup.

To encode the $\mathrm{BC}$ decomposition into an integer linear program, we use the linear encoding of variables domains [16]. We introduce literals $c_{i j}$ for the truth of $X_{i} \leq j$, and the channeling inequalities of the form $c_{i(l-1)}+1-c_{i u}+M_{l u} \geq 1$. We again add the redundant constraint $\sum_{i=1}^{n^{2}} M_{i i}=M_{1, n^{2}}$. Finally, we post constraints (6) as lazy constraints in CLPEX. Lazy constraints are constraints that are not expected to be violated when they are omitted. These constraints are not taken into account in the relaxation of the problem and are only included when they violate an integral solution.

Table 1. Backtracks and rumtime (in seconds) to solve the dominating set problem for the Queen's graph.

\begin{tabular}{|l|l||rr|rr|rr|rr|}
\hline$n$ & $N$ & \multicolumn{2}{|c|}{ Occs } & \multicolumn{2}{|c|}{ Pyramid ${ }_{B C}$} & \multicolumn{2}{|c|}{ Occs } & \multicolumn{2}{|c|}{ Pyramid $_{B C}^{\text {CPLEX }}$} \\
\hline \multicolumn{2}{|l|}{} & backtracks & time & backtracks & time & backtracks & time & backtracks & time \\
\hline 5 & 3 & 34 & 0.01 & 7 & $\mathbf{0 . 0 0}$ & 1 & 0.05 & 3 & 0.4 \\
6 & 3 & 540 & 0.16 & 118 & $\mathbf{0 . 0 3}$ & 2 & 0.16 & 183 & 9.6 \\
7 & 4 & 195,212 & 84.50 & 83,731 & $\mathbf{1 5 . 4 9}$ & 130,010 & 1802.49 & 63 & 15.8 \\
8 & 5 & 390,717 & 255.64 & 256,582 & 58.42 & 24,588 & 585.07 & 30 & $\mathbf{4 1 . 2 8}$ \\
\hline
\end{tabular}

Results of our experiments are presented in Table 1. Our BC decomposition performs better than the Occs decomposition, both in runtime and in number of backtracks needed by Ilog Solver or CPLEX. CPLEX is slower per node than Ilog Solver. However, CPLEX usually requires fewer backtracks compared to ILOG Solver. Interestingly CPLEX performs well with the $\mathrm{BC}$ decomposition. The time to explore each node is large, reflecting the size of decomposition, but the number of search nodes explored is small. We conjecture that integer linear programming methods like CPLEX will perform in a similar way with other decompositions of global constraints which do not hinder propagation (e.g. the decompositions we have proposed for ALL-DIFFERENT and GCC). Finally, the best results here are comparable with those for the ATMOstNVALUE bounds consistency propagator in [13].

\section{Other related work}

Bessiere et al. consider a number of different methods to compute a lower bound on the number of values used by a set of variables [13]. One method is based on a simple linear relaxation of the minimum hitting set problem. This gives a propagation algorithm that achieves a level of consistency strictly stronger than bound consistency on the NVALUE constraint. Cheaper approximations are also proposed based on greedy heuristics and an approximation for the independence number of the interval graph due to Turán. Decompositions have been given for a number of other global constraints. For example, Beldiceanu et al. identify conditions under which global constraints specified as automata can be decomposed into signature and transition constraints without hindering 
propagation [17]. As a second example, many global constraints can be decomposed using ROOTS and RANGE which can themselves be propagated effectively using simple decompositions [18]. As a third example, the REGULAR and CFG constraints can be decomposed without hindering propagation $[19,20]$. As a fourth example, decompositions of the SEQUENCE constraint have been shown to be effective [21]. Most recently, we demonstrated that the ALL-DIFFERENT and GCC constraint can be decomposed into simple primitive constraints without hindering bound consistency propagation [5]. These decompositions also introduced variables to count variables using values in an interval. For example, the decomposition of ALL-DIFFERENT ensures that no interval has more variables taking values in the interval than the number of values in the interval. Using a circuit complexity lower bound, we also proved that there is no polynomial sized SAT decomposition of the ALL-DIFFERENT constraint (and therefore of its generalizations like NVALUE) on which unit propagation achieves domain consistency [6]. Our use of "pyramid" variables is similar to the use of the "partial sums" variables in the encoding of the SEQUENCE constraint in [21]. This is related to the cumulative sums computed in [22].

\section{Conclusions}

We have studied a number of decompositions of the NVALUE constraint. We have shown that a simple decomposition can simulate the bound consistency propagator for NVALUE [12] with comparable time complexity but with a much greater space complexity. This supports the conclusion that the benefit of a global propagator may often not be in saving time but in saving space. Our other theoretical contribution is to show the first range consistency algorithm for NVALUE, that runs in $O\left(n d^{3}\right)$ time and $O\left(n d^{2}\right)$ space. These results are largely interesting from a theoretical perspective. They help us understand the globality of global constraints. They highlight that saving space may be one of the important advantages provided by propagators for global constraints. We have seen that the space complexity of decompositions of many propagators equals the worst case time complexity (e.g. for the ALL-Different, GCC, AMONG, LEX, Regular, CFG and SEQuence constraints). For global constraints like Regular, the space complexity of the decompositions does not appear to be that problematic. However, for global constraints like NVALUE, the space complexity of the decompositions is onerous. This space complexity seems hard to avoid. For example, consider encodings into satisfiability and unit propagation as our inference method. As unit propagation is linear in time in the size of the encoding, it is somewhat inevitable that the size of any encoding is the same as the worst-case time complexity of any propagator that is being simulated. One other benefit of these decompositions is that they help us explore the interface between constraint and integer linear programming. For example, we saw that an integer programming solver performed relatively well with these decompositions.

Acknowledgements. NICTA is funded by the Department of Broadband, Communications and the Digital Economy, and the ARC. Christian Bessiere is supported by ANR project ANR-06-BLAN-0383-02, and George Katsirelos by ANR UNLOC project: ANR 08-BLAN-0289-01. We thank Lanbo Zheng for experimental help. 


\section{References}

1. Régin, J.C.: A filtering algorithm for constraints of difference in CSPs. In: Proc. of the 12th National Conf. on AI, AAAI (1994) 362-367

2. Leconte, M.: A bounds-based reduction scheme for constraints of difference. In: Proc. of 2nd Int. Workshop on Constraint-based Reasoning (Constraint-96). (1996)

3. Puget, J.: A fast algorithm for the bound consistency of alldiff constraints. In: 15th National Conf. on Artificial Intelligence, AAAI (1998) 359-366

4. Bessiere, C., Hentenryck, P.V.: To be or not to be ... a global constraint. In: Proc. of 9th Int Conf. on Principles and Practice of Constraint Programming (CP 2003), (2003) 789-794

5. Bessiere, C., Katsirelos, G., Narodytska, N., Quimper, C.G., Walsh, T.: Decompositions of all different, global cardinality and related constraints. In: Proc. of 21st IJCAI, (2009) $419-424$

6. Bessiere, C., Katsirelos, G., Narodytska, N., Walsh, T.: Circuit complexity and decompositions of global constraints. In: Proc. of 21st IJCAI, (2009) 412-418

7. Bessiere, C., Hebrard, E., Hnich, B., Walsh, T.: The complexity of global constraints. In: Proc. of the 19th National Conf. on AI, AAAI (2004)

8. Pachet, F., Roy, P.: Automatic generation of music programs. In: Proc. of 5th Int. Conf. on Principles and Practice of Constraint Programming (CP99), (1999) 331-345

9. Bessiere, C., Hebrard, E., Hnich, B., Kiziltan, Z., Walsh, T.: Filtering algorithms for the NVALUE constraint. In: Proc. 2nd Int. Conf. on Integration of AI and OR Techniques in Constraint Programming for Combinatorial Optimization Problems. (2005)

10. Bessiere, C., Hebrard, E., Hnich, B., Walsh, T.: The complexity of global constraints. Constraints 12 (2007) 239-259

11. Bessiere, C., Hebrard, E., Hnich, B., Kiziltan, Z., Quimper, C.G., Walsh, T.: The parameterized complexity of global constraints. In: Proc. of the 23rd National Conf. on AI, AAAI (2008) 235-240

12. Beldiceanu, N.: Pruning for the minimum constraint family and for the number of distinct values constraint family. In: Proc. of 7th Int. Conf. on Principles and Practice of Constraint Programming (CP2001), (2001) 211-224

13. Bessiere, C., Hebrard, E., Hnich, B., Kiziltan, Z., Walsh, T.: Filtering algorithms for the NVALUE constraint. Constraints 11 (2006) 271-293

14. Stergiou, K., Walsh, T.: The difference all-difference makes. In: Proc. of 16th IJCAI, (1999)

15. Östergård, P., Weakley, W.: Values of domination numbers of the queen's graph. The Electronic Journal of Combinatorics 8 (2001)

16. Ohrimenko, O., Stuckey, P., Codish, M.: Propagation = lazy clause generation. In: Proc. of 13th Int. Conf. on Principles and Practice of Constraint Programming (CP-2007), (2007)

17. Beldiceanu, N., Carlsson, M., Debruyne, R., Petit, T.: Reformulation of Global Constraints Based on Constraints Checkers. Constraints 10 (2005) 339-362

18. Bessiere, C., Hebrard, E., Hnich, B., Kiziltan, Z., Walsh, T.: The range and roots constraints: Specifying counting and occurrence problems. In: Proc. of 19th IJCAI, (2005) 60-65

19. Quimper, C.G., Walsh, T.: Global grammar constraints. In: Proc. of 12th Int. Conf. on Principles and Practice of Constraint Programming (CP-2006), (2006)

20. Quimper, C.G., Walsh, T.: Decomposing global grammar constraints. In: Proc. of 13th Int. Conf. on Principles and Practice of Constraint Programming (CP-2007), (2007)

21. Brand, S., Narodytska, N., Quimper, C.G., Stuckey, P., Walsh, T.: Encodings of the Sequence Constraint. In: Proc. of 13th Int. Conf. on Principles and Practice of Constraint Programming (CP-2007), (2007)

22. van Hoeve, W.J., Pesant, G., Rousseau, L.M., Sabharwal, A.: Revisiting the Sequence Constraint. In: Proceedings of the 12th Int. Conf. on Principles and Practice of Constraint Programming (CP-2006), (2006) 